\title{
Peptides: A Package for Data Mining of Antimicrobial Peptides
}

by Daniel Osorio, Paola Rondón-Villarreal and Rodrigo Torres

\begin{abstract}
Antimicrobial peptides (AMP) are a promising source of antibiotics with a broad spectrum activity against bacteria and low incidence of developing resistance. The mechanism by which an AMP executes its function depends on a set of computable physicochemical properties from the amino acid sequence. The Peptides package was designed to allow the quick and easy computation of ten structural characteristics own of the antimicrobial peptides, with the aim of generating data to increase the accuracy in classification and design of new amino acid sequences. Moreover, the options to read and plot XVG output files from GROMACS molecular dynamics package are included.
\end{abstract}

\section{Introduction}

Antimicrobial peptides are a promising source of antibiotics with a broad spectrum activity against bacteria and low incidence of developing resistance (Hancock, 2001). Multiple research has concluded that the natural biological activities of these peptides are coordinated by a sophisticated modulation of the hydrophobicity, amphipathicity, positive charge and a reduction in the hydrophobic moment (Yeaman and Yount, 2003; Fjell et al., 2012; Matsuzaki, 2009). Additionally to these four properties there are other descriptors that used in conjunction can provide useful information in the classification and design of antimicrobial peptides (Boman, 2003; Wang et al., 2009; Thomas et al., 2010; Piotto et al., 2012).

The computation of these properties for antimicrobial peptides is available free of charge through various web or native applications as ExPASy-protparam (Gasteiger et al., 2005), EMBOSS-pepstats (Rice et al., 2000), BioPerl (Stajich et al., 2002), CAMP (Thomas et al., 2010) and APD (Wang et al., 2009) databases. However, no application allows the computation of all properties. Some of these services allow only the computation of some properties for a sequence at a time, and have no option for downloading and handling this information in editable files. Others allow the calculation of more than one sequence, but they write an output file for each one of them, which makes it difficult to handle and analyse the data.

Taking the advantage of handling data, vectors and tables provided by $\mathrm{R}$, we introduce the Peptides package. It allows quick and easy computation of ten structural characteristics own of the antimicrobial peptides (length, amino-acid composition, net charge, aliphatic index, molecular weight, isoelectric point, hydrophobic moment, potential peptide interaction index, instability index and GRAVY hydrophobicity index) in a single application. The Peptides package was designed with the aim of generating data to increase the accuracy in the classification process of new amino acid sequences. In addition, the option to read and plot XVG output files from GROMACS molecular dynamics package was included.

In this work we describe the computation of structural properties of the Lasiocepsin peptide (GLPRKILCAIAKKKGKCKGPLKLVCKC) using the Peptides package, step by step as an example. It is an alpha-helical antimicrobial peptide derived from the venom of eusocial bee Lasioglossum laticeps, identified in the Protein Data Bank with the 2MBD code (Monincová et al., 2012). Moreover, an example of a classification using a data set of 23 variables computed for 100 peptides through Peptides is performed. It was found that using this data set for classification through linear discriminant analysis and classification-regression trees allows to classify antimicrobial peptides with an accuracy of $95 \%$ and $85 \%$, respectively.

\section{Installation and functions}

Peptides includes thirteen functions and is available for download and installation from CRAN, the Comprehensive R Archive Network. To install it, just type:

$>$ install.packages("Peptides")
$>$ library(Peptides)

The Peptides package requires $\mathrm{R}$ version 1.2.2 or higher. Development releases of the package are available on the GitHub repository http://github.com/dosorio/peptides. 


\section{Number of amino acids}

As all proteins, the antimicrobial peptides are formed by linear chains of small residues known as amino acids attached to each other by peptide bonds. Antimicrobial peptides are characterized by a short length, they generally comprise less than 50 amino acids. This property minimizes the probability of being degraded by bacterial proteases (Kim et al., 2013). The function lengthpep counts the number of amino acids in a sequence and returns a vector with the count for each peptide used as argument.

$>$ lengthpep $($ seq $=$ "GLPRKILCAIAKKKGKCKGPLKLVCKC")

[1] 27

\section{Molecular weight}

The molecular weight is the sum of the masses of each atom constituting a molecule. The molecular weight is directly related to the length of the amino acid sequence and is expressed in units called daltons (Da). Antimicrobial peptides due to its short length are characterized by a molecular weight $<10 \mathrm{kDa}(10000 \mathrm{Da})$. In Peptides the function mw computes the molecular weight using the same formulas and weights as ExPASy's "compute pI/mw" tool (Gasteiger et al., 2005).

$>\mathrm{mw}(\mathrm{seq}=$ "GLPRKILCAIAKKKGKCKGPLKLVCKC")

[1] 2897.787

\section{Amino acid composition}

Amino acids are zwitterionic molecules with an amine and a carboxyl group present in their structure. Some amino acids possess side chains with specific properties that allow grouping them in different ways. The aacomp function classifies amino acids based on their size, side chains, hydrophobicity, charge and their response to $\mathrm{pH} 7$ following the categories listed in Table 1. The output is a matrix with the number and percentage of amino acids of a particular class.

\begin{tabular}{ll}
\hline Class & Amino acids \\
\hline Tiny & A C G T \\
Small & A B C D G N S T V \\
Aliphatic & A I L V \\
Aromatic & F H Y \\
Non-polar & A C F I L M P V W Y \\
Polar & D E H K N R T Z \\
Charged & B D E H R R \\
Basic & H K R \\
Acidic & B D E Z \\
\hline
\end{tabular}

Table 1: Classification of the amino acids according to the properties of size and side chain.

Antimicrobial peptides are amphipathic (with similar proportions of polar and non-polar amino acids) and charged molecules. In Peptides the amino acid composition can be computed using the function aacomp.

$\begin{array}{lrr}\text { >acomp }(\mathrm{seq}= & \text { "GLPRKILCAIAKKKGKCKGPLKLVCKC") } \\ & \text { Number } & \text { Mole\% } \\ \text { Tiny } & 9 & 33.333 \\ \text { Small } & 12 & 44.444 \\ \text { Aliphatic } & 9 & 33.333 \\ \text { Aromatic } & 0 & 0.000 \\ \text { NonPolar } & 18 & 66.667 \\ \text { Polar } & 9 & 33.333 \\ \text { Charged } & 9 & 33.333 \\ \text { Basic } & 9 & 33.333 \\ \text { Acidic } & 0 & 0.000\end{array}$




\section{Net charge}

Some side chains of certain amino acids can confer electric charge to the proteins under certain $\mathrm{pH}$ values. The sum of the charges of each of the amino acids is called net charge. Antimicrobial peptides have a positive net charge (of at least +2 ) at $\mathrm{pH} 7$, which provides binding specificity to the negatively charged bacterial membranes through electrostatic interactions (Yeaman and Yount, 2003).

The charge function compute the net charge using Equation 1, a variation of the Henderson Hasselbalch equation proposed by Moore (1985) wherein $N$ are the number, $j$ and $i$ index represent the acidic (Aspartic Acid, Glutamic Acid, Cysteine and Tyrosine) and basic (Arginine, Lysine, and Histidine) functional groups of amino acids, respectively.

The net charge of a protein can be calculated specifying the $\mathrm{pH}$ value and one of the nine $\mathrm{pKa}$ scales availables (Bjellqvist, Dawson, EMBOSS, Lehninger, Murray, Rodwell, Sillero, Solomon or Stryer).

$$
\text { charge }=\sum_{i} N_{i} \frac{+1}{1+10^{+\left(p H-p K_{a i}\right)}}+\sum_{j} N_{j} \frac{-1}{1+10^{-\left(p H-p K_{a j}\right)}}
$$

$>\operatorname{charge}(\mathrm{seq}=$ "GLPRKILCAIAKKKGKCKGPLKLVCKC", $\mathrm{pH}=7$, pKscale $=$ "EMBOSS")

[1] 8.85201

\section{Isoelectric point}

The isoelectric point (pI) is the $\mathrm{pH}$ at which the net charge of the protein is equal to 0 . It is a variable that affects the solubility of the peptides under certain conditions of $\mathrm{pH}$. When the $\mathrm{pH}$ of the solvent is equal to the $\mathrm{pI}$ of the protein, it tends to precipitate and loose its biological function. Antimicrobial peptides have an isoelectric point close to 10 (Torrent et al., 2011), which is very similar to soap or detergent and consistent with the proposed mechanisms of action for these peptides.

The calculation of the isoelectric point of a peptide may be performed through the function $\mathrm{pI}$ specifying one of the nine pKa scales available (Bjellqvist, Dawson, EMBOSS, Lehninger, Murray, Rodwell, Sillero, Solomon or Stryer).

$>$ pI $($ seq $=$ "GLPRKILCAIAKKKGKCKGPLKLVCKC", pKscale $=$ "EMBOSS")

[1] 10.801

\section{Aliphatic index}

It has been suggested that the aliphatic amino acids (A, I, L and V) are responsible for the thermal stability of proteins. The aliphatic index was proposed by Ikai (1980) and evaluates the thermostability of proteins based on the percentage of each of the aliphatic amino acids that build up proteins. This index is computed using Equation 2 wherein $X_{A}, X_{V}, X_{I}$ and $X_{L}$ are the mole percent $(100 \mathrm{x}$ mole fraction) of Alanine, Valine, Isoleucine and Leucine respectively.

$$
A I=X_{A}+2.9 X_{V}+3.9\left(X_{I}+X_{L}\right)
$$

Antimicrobial peptides tend to be more thermostable than proteins in general. For the calculation of the aliphatic index, the function aindex was included.

$>$ aindex (seq $=$ "GLPRKILCAIAKKKGKCKGPLKLVCKC")

[1] 104.8148

\section{Instability index}

The instability index was proposed by Guruprasad et al. (1990). This index predicts the stability of a protein based on its amino acid composition. It is calculated according to Equation 3 where $L$ is equal to the length of the amino acid sequence; $X_{i} Y_{i}$ is a dipeptide and DIWV is the dipeptide weight value on amino acid sequence of stable proteins.

$$
I I=\left(\frac{10}{L} \sum_{i=10}^{L-1} \operatorname{DIW} V_{\left(X_{i} Y_{i}+1\right)}\right)
$$

Despite their short length (variable that this function penalizes), antimicrobial peptides tend to be considered stable with index values less than 40 . The instability index can be calculated using the function instaindex incorporated in Peptides. 
$>$ instaindex $($ seq $=$ "GLPRKILCAIAKKKGKCKGPLKLVCKC" $)$

[1] 2.237037

\section{Boman index}

The potential protein interaction index was proposed by Boman (2003) as an easy way to differentiate the action mechanism of hormones (protein-protein) and antimicrobial peptides (protein-membrane) through this index. It is calculated using Equation 4 by adding each amino acid solubilities divided by the sequence length. This function predicts the potential peptide interaction with another protein.

$$
B_{\text {index }}=\frac{\sum_{i=1}^{N} S_{i}}{N}
$$

During its mechanism of action, antimicrobial peptides tend to not interact with other proteins (the proposed mechanism of action is based on the interaction with membranes), so the values for the Boman index are usually negative or nearby to 0 . To calculate the Boman index, the boman function is included within Peptides.

$>\operatorname{boman}($ seq $=$ "GLPRKILCAIAKKKGKCKGPLKLVCKC")

[1] 0.5259259

\section{Hydrophobicity index}

The hydrophobicity is an important stabilization force in protein folding; this force changes depending on the solvent in which the protein is found. It is considered the driving force of the peptide to the core of the membrane. The hydrophobicity index is calculated following the Equation 5, adding the hydrophobicity of individual amino acids and dividing this value by the length of the sequence. Highly expected transmembrane peptides generally have higher hydrophobicity values than 0.5 using Eisenberg scale.

$$
H=\frac{\sum_{i=1}^{N} H_{i}}{N}
$$

Peptides includes thirty-eight scales of hydrophobicity (Aboderin, AbrahamLeo, Argos, BlackMould, BullBreese, Casari, Chothia, Cid, Cowan3.4, Cowan7.5, Eisenberg, Engelman, Fasman, Fauchere, Goldsack, Guy, HoppWoods, Janin, Jones, Juretic, Kidera, Kuhn, KyteDoolittle, Levitt, Manavalan, Miyazawa, Parker, Ponnuswamy, Prabhakaran, Rao, Rose, Roseman, Sweet, Tanford, Welling, Wilson, Wolfenden or Zimmerman) obtained from various sources (Gasteiger et al., 2005; Kawashima et al., 2008).

$>$ hydrophobicity (seq = "GLPRKILCAIAKKKGKCKGPLKLVCKC", scale = "Eisenberg")

[1] -0.08777778

\section{Hydrophobic moment index}

The hydrophobic moment was proposed by Eisenberg et al. (1982), as a quantitative measure of the amphiphilicity perpendicular to the axis of any periodic peptide structure. To calculate the hydrophobic moment, the function hmoment was included. The hydrophobic moment is computed according to Equation 6, using the standardized Eisenberg (1984) scale, windows (fragment of sequence) of eleven amino acids and specifying the rotational angle at which it should be calculated. Highly expected transmembrane peptides generally have lower hydrophobic moment values than 0.2 .

$$
\mu H=\left\{\left[\sum_{n=1}^{N} H_{n} \sin (\delta n)\right]^{2}+\left[\sum_{n=1}^{N} H_{n} \cos (\delta n)\right]^{2}\right\}^{\frac{1}{2}}
$$

$>\operatorname{hmoment}(\mathrm{seq}=$ "GLPRKILCAIAKKKGKCKGPLKLVCKC", angle $=100$, window $=11)$

[1] 0.6170697

$>\operatorname{hmoment}(\mathrm{seq}=$ "GLPRKILCAIAKKKGKCKGPLKLVCKC", angle $=160$, window $=11)$

[1] 0.4617153 


\section{Membrane position}

Eisenberg et al. (1982) found a correlation between hydrophobicity and hydrophobic moment that defines the protein section as globular, transmembrane or superficial. The function calculates the hydrophobicity $(\mathrm{H})$ and hydrophobic moment $(\mu \mathrm{H})$ based on the standardized scale of Eisenberg (1984) using windows of 11 amino acids for calculate the theoretical fragment type.

$>\operatorname{membpos}(\mathrm{seq}=$ "GLPRKILCAIAKKKGKCKGPLKLVCKC", angle $=100)$

$\begin{array}{llrrr} & \text { Pep } & \text { H } & \text { uH } & \text { MembPos } \\ 1 & \text { GLPRKILCAIA } & 0.271 & 0.469 & \text { Surface } \\ 2 & \text { LPRKILCAIAK } & 0.091 & 0.617 & \text { Surface } \\ 3 & \text { PRKILCAIAKK } & -0.142 & 0.520 & \text { Globular } \\ 4 & \text { RKILCAIAKKK } & -0.289 & 0.401 & \text { Globular } \\ 5 & \text { KILCAIAKKKG } & -0.015 & 0.325 & \text { Globular } \\ 6 & \text { ILCAIAKKKGK } & -0.015 & 0.319 & \text { Globular } \\ 7 & \text { LCAIAKKKGKC } & -0.115 & 0.339 & \text { Globular } \\ 8 & \text { CAIAKKKGKCK } & -0.347 & 0.115 & \text { Globular } \\ 9 & \text { AIAKKKGKCKG } & -0.330 & 0.096 & \text { Globular } \\ 10 & \text { IAKKKGKCKGP } & -0.375 & 0.141 & \text { Globular } \\ 11 & \text { AKKKGKCKGPL } & -0.405 & 0.161 & \text { Globular } \\ 12 & \text { KKKGKCKGPLK } & -0.597 & 0.110 & \text { Globular } \\ 13 & \text { KKGKCKGPLKL } & -0.365 & 0.156 & \text { Globular } \\ 14 & \text { KGKCKGPLKLV } & -0.130 & 0.310 & \text { Globular } \\ 15 & \text { GKCKGPLKLVC } & 0.033 & 0.257 & \text { Globular } \\ 16 & \text { KCKGPLKLVCK } & -0.147 & 0.426 & \text { Globular } \\ 17 & \text { CKGPLKLVCKC } & 0.015 & 0.487 & \text { Globular }\end{array}$

\section{GROMACS files}

Molecular dynamics is a source of in-silico biophysical data that contribute to the design, classification and testing of antimicrobial peptides. GROMACS (GROningen MAchine for Chemical Simulations) is a molecular dynamics package designed for simulations of proteins, lipids and nucleic acids. It is free, open source software released under the GNU General Public License.

The file format used by GROMACS is XVG. This format can be displayed in graphical form through the GRACE program on UNIX/LINUX systems and the GNUPlot program on Windows. XVG files are plain text files containing tabular data separated by tabulators and two types of comments which contain data labels. Although manual editing is possible, this is not a viable option when working with multiple files of this type.

For ease of reading, information management and data plotting, the functions read.xvg and plot.xvg were incorporated. An example of how to read and plot the absolute distance between the center of mass of an antimicrobial peptide with respect to a POPG (1-palmitoyl-2-oleoyl-sn-glycero-3phosphoglycerol) pure lipid bilayer is presented below (Figure 1).

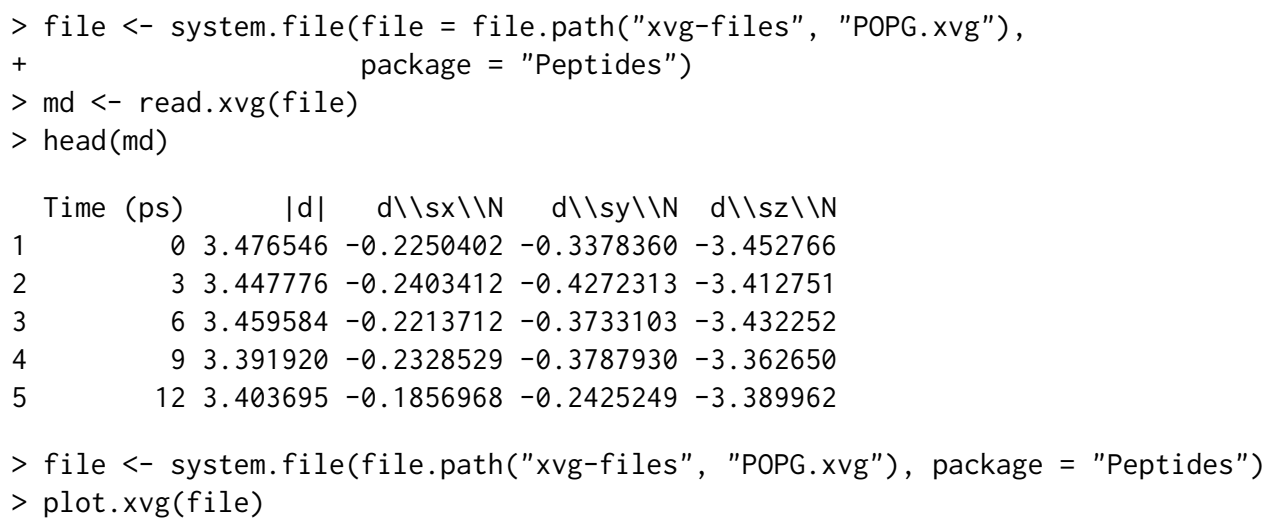

\section{Using data in the classification process}

Altogether the data produced in Peptides are a useful source of specifications allowing classification of antimicrobial peptides and their differentiation from other peptides such as hormones. An example 
Distance

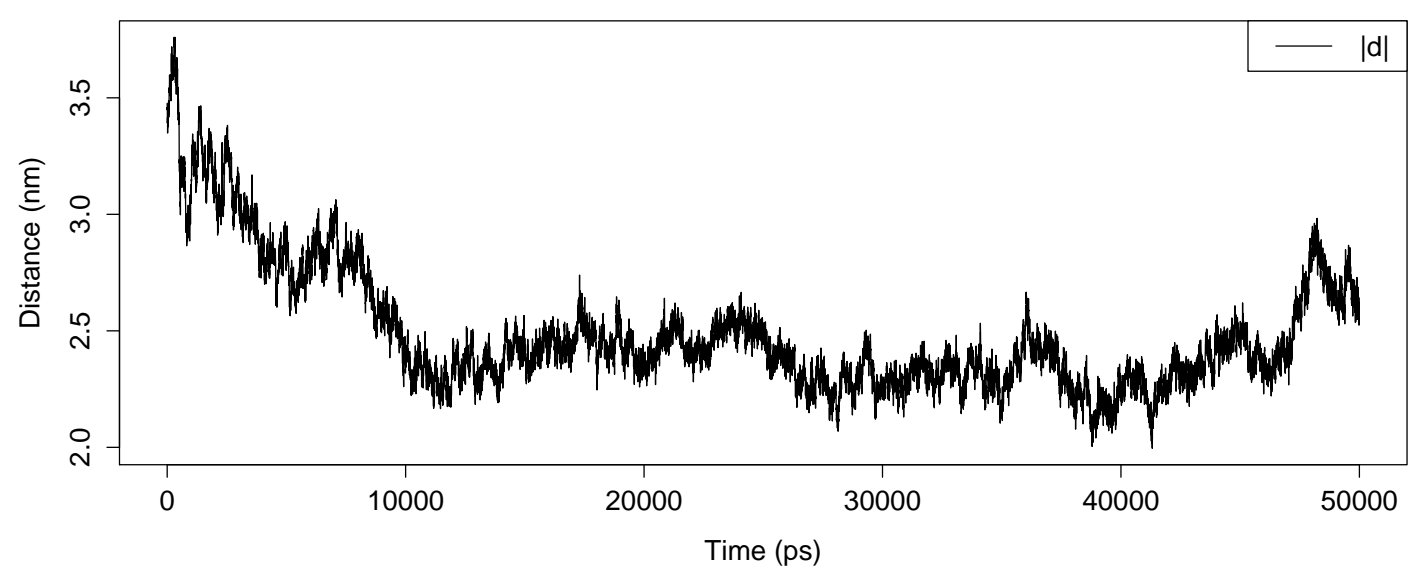

Figure 1: Absolute distance between the center of mass of an antimicrobial peptide with respect to a POPG pure lipid bilayer.

of classification using linear discriminant analysis and classification-regression trees through the caret package (Kuhn, 2014) is shown.

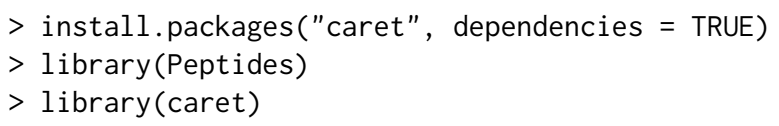

The data set was calculated using all the functions included in Peptides. It is available in the data. frame with 100 observations and 23 variables called pepdata. It includes the physicochemical properties and calculable indices for 50 antimicrobial (group $=1$ ) and 50 non-antimicrobial (group =0) peptides downloaded from the Protein Data Bank (Bernstein et al., 1978) and the APD database (Wang et al., 2009) respectively.

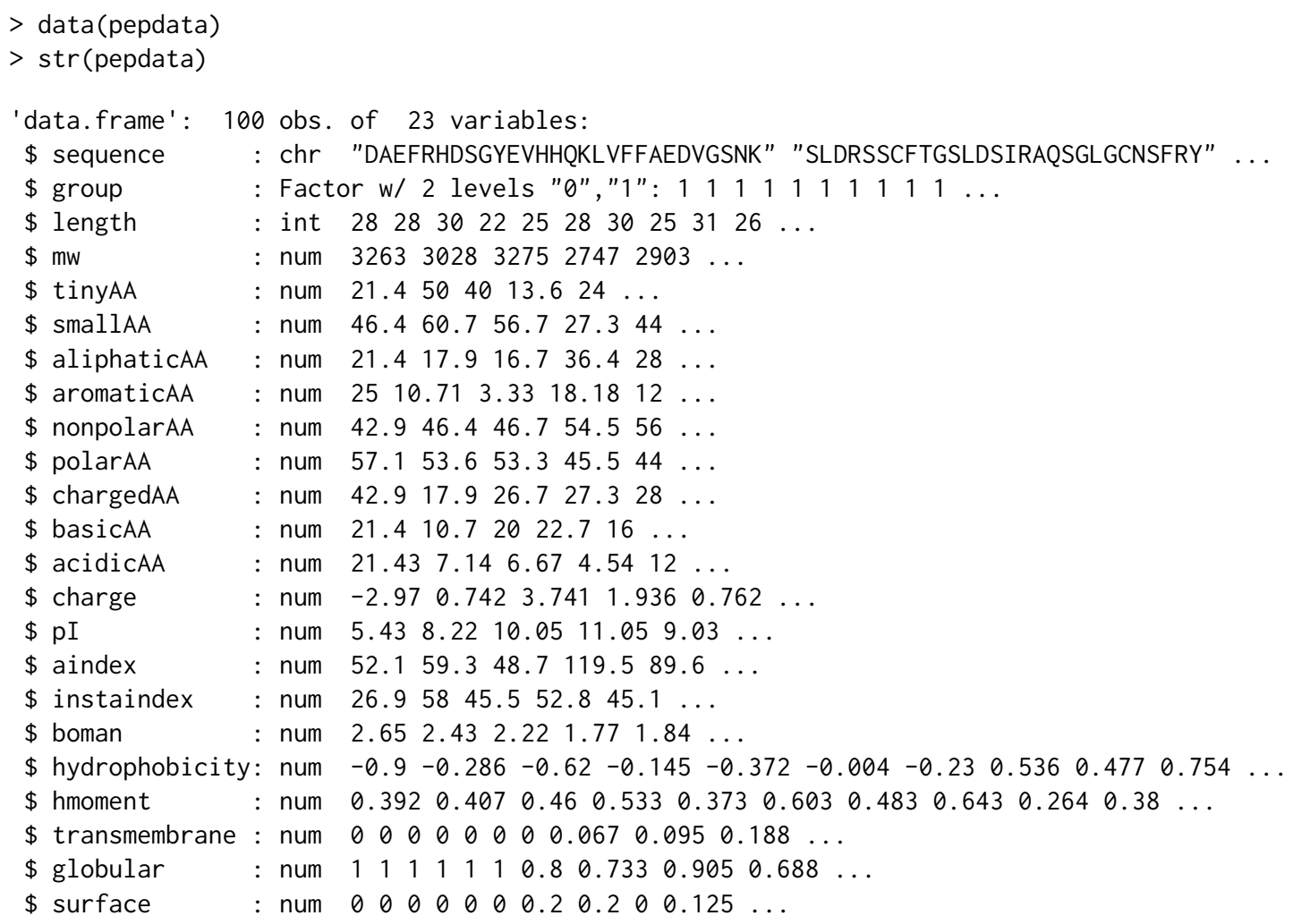

The training and testing data sets were created with the following commands. 


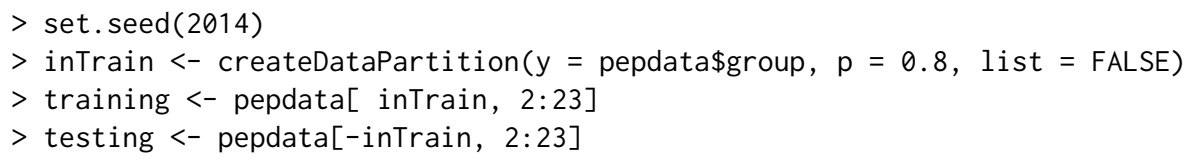

To evaluate the potential of these data, the best CART model was selected by a $K$-fold cross-validation loop.

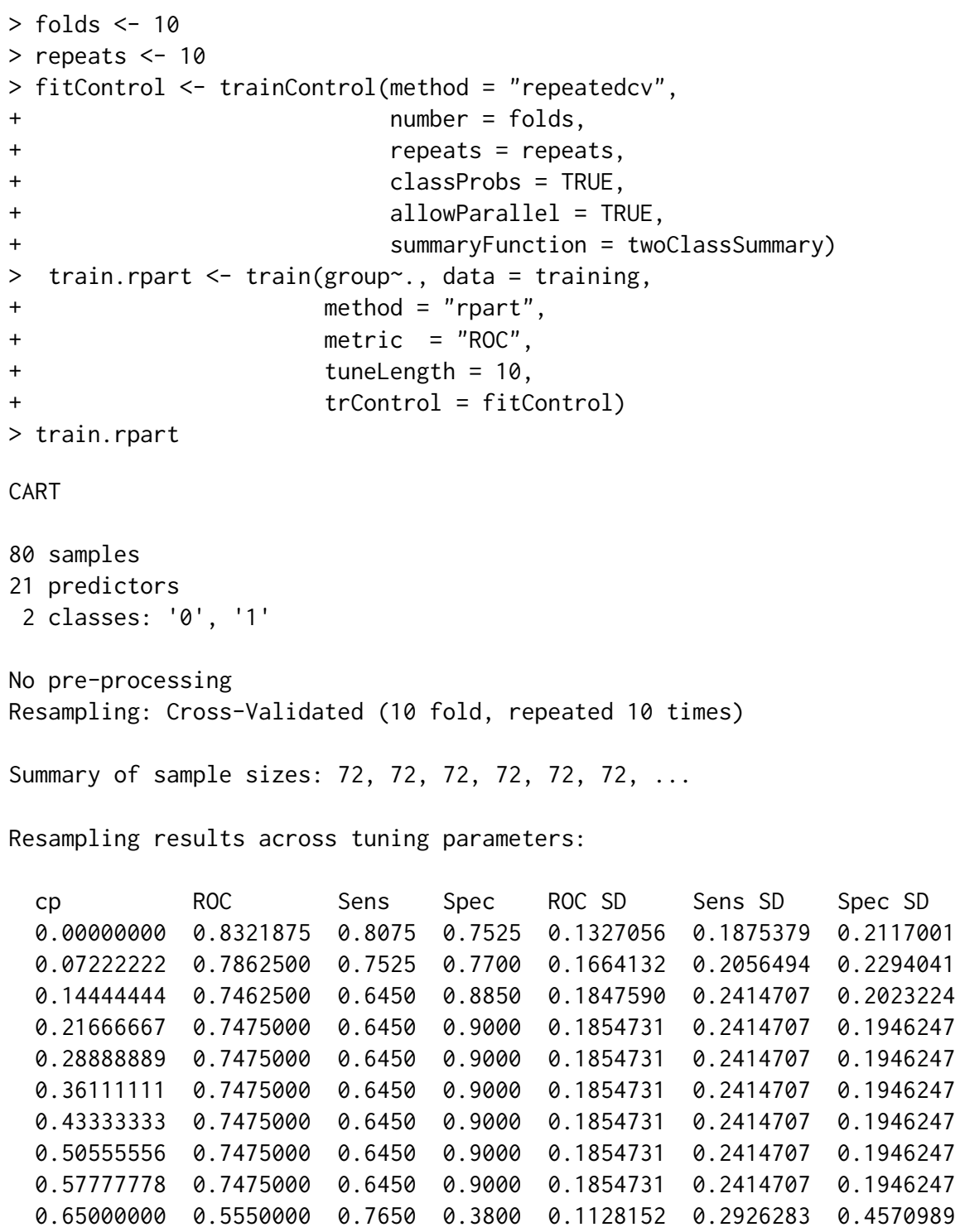

ROC was used to select the optimal model using the largest value.

The final value used for the model was $c p=0$.

$>\operatorname{plot}($ train.rpart)

To evaluate the accuracy of the classifier a prediction with testing data was performed.

$>$ pred.rpart <- predict(train.rpart, newdata = testing)

$>$ pred.rpart

[1] 1000000000000001110011111111

Levels: 01

Classification-regression trees using this data set showed an accuracy of $85 \%$, a sensitivity of $80 \%$ and a specificity of $90 \%$ to differentiate antimicrobial from non-antimicrobial peptides.

$>$ confusionMatrix (data $=$ pred.rpart, testing\$group, positive $=" 1 "$ ) 


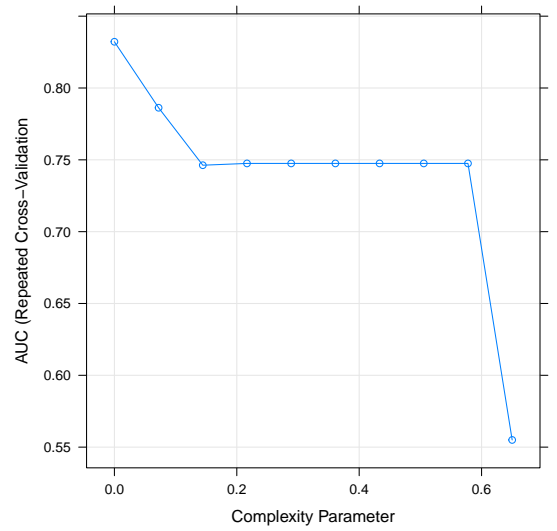

charge 1.321

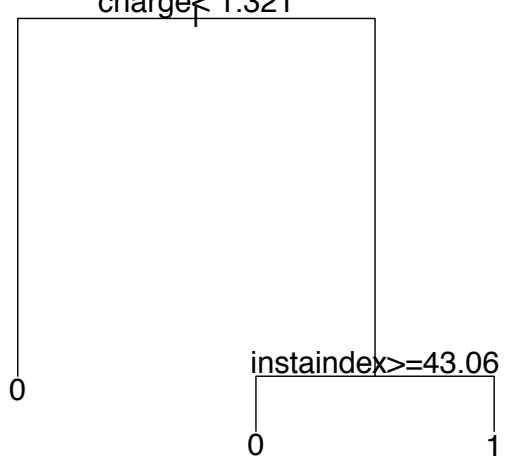

Figure 2: plot (train.rpart) shows the relationship between the complex parameter and the resampled estimate area under the ROC curve used to find the best model. plot (tree) shows the rules to classify the antimicrobial peptides of the Pepdata data set.

Confusion Matrix and Statistics

Reference

Prediction 01

092

118

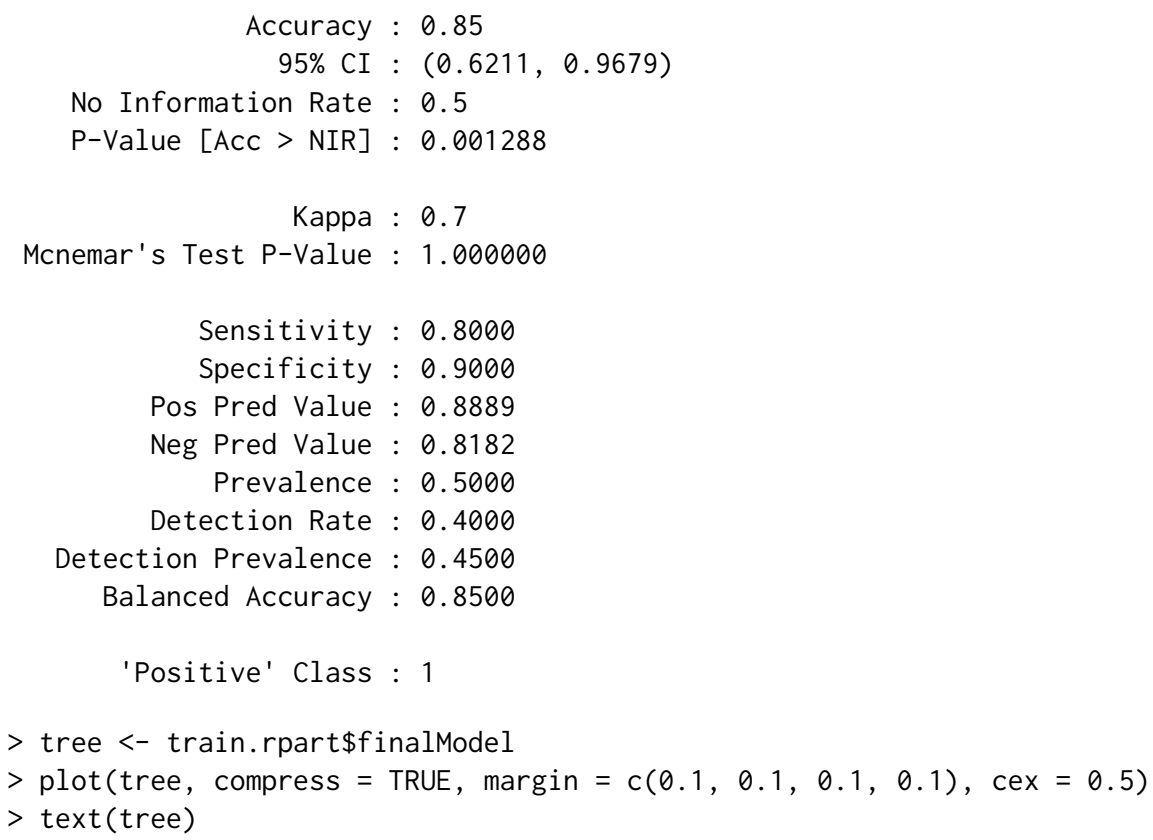

For this data set, the resulting classification-regression tree is based on characteristic properties of antimicrobial peptides such as charge and instability index (Figure 2).

The same process was conducted to evaluate the data potential using a linear discriminant analysis.

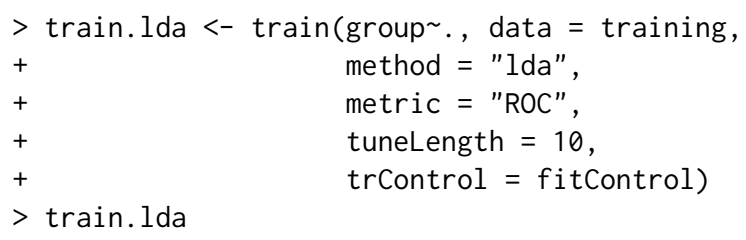

Linear Discriminant Analysis

80 samples 


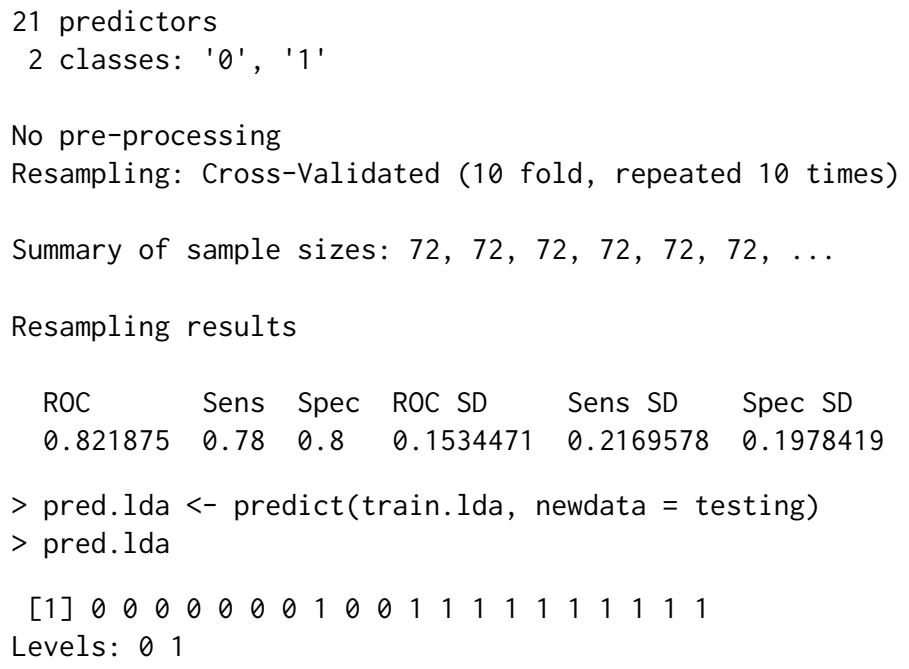

The linear discriminant analysis using this data set showed an accuracy of $95 \%$, a sensitivity of $100 \%$ and a specificity of $90 \%$ to differentiate antimicrobial from non-antimicrobial peptides.

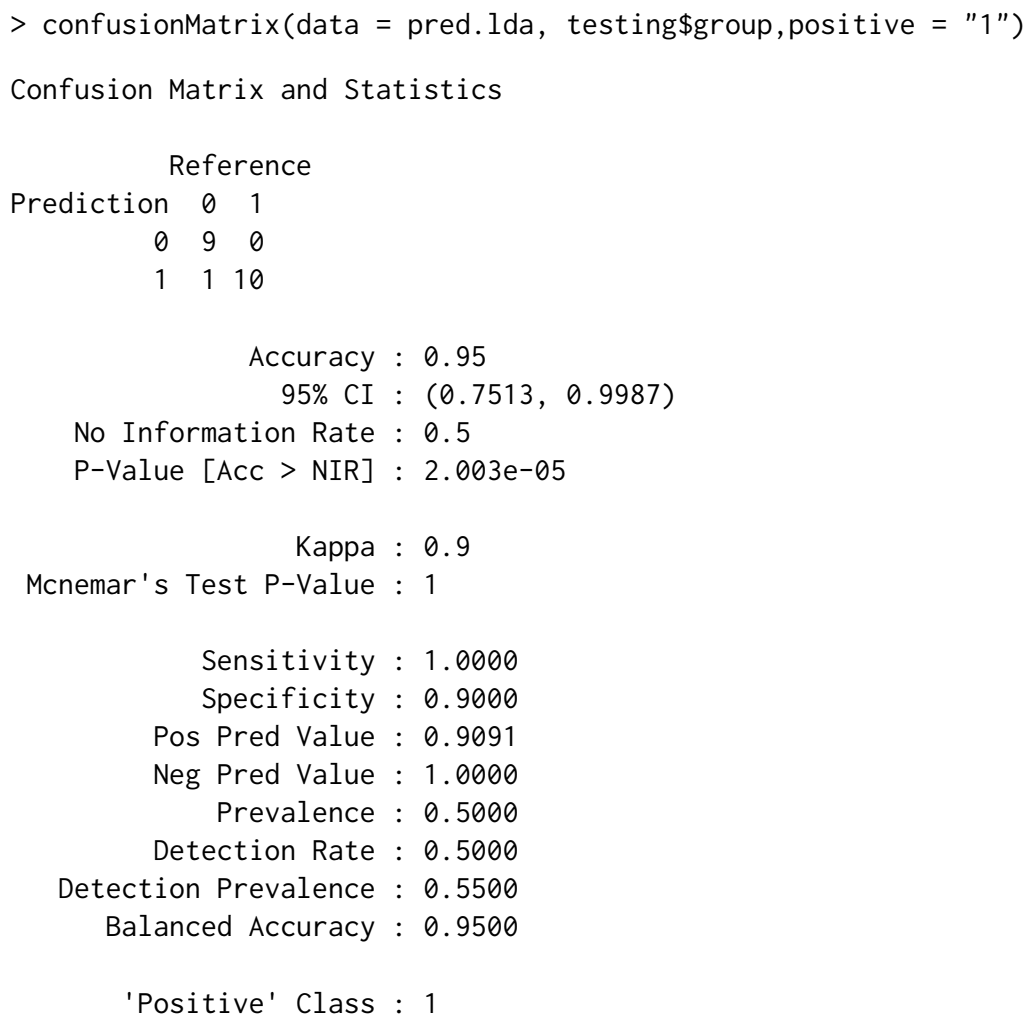

\section{Summary}

We introduced the Peptides package to calculate physicochemical properties and indices from amino acids sequences of peptides and proteins. We also showed how the package's functions can be used to produce useful data in classification-regression processes and handle the output files from the GROMACS molecular dynamics package. The authors are currently working on extending the package by the addition of new biological functions such as peptide flexibility (Huang and Nau, 2003), molecular lipophilicity potential (Gaillard et al., 1994), cell penetrating parameter (Holton et al., 2013) and free energy of binding (Hessa et al., 2007).

\section{Acknowledgements}

This project corresponds to a part of the first author's undergraduate thesis. We thank COLCIENCIAS from Colombia for the fellowship to PR and to the anonymous reviewers for their helpful comments 
and suggestions.

\section{Bibliography}

F. C. Bernstein, T. F. Koetzle, G. J. Williams, E. F. Meyer, M. D. Brice, J. R. Rodgers, O. Kennard, T. Shimanouchi, and M. Tasumi. The Protein Data Bank: A computer-based archival file for macromolecular structures. Archives of Biochemistry and Biophysics, 185(2):584-591, 1978. [p9]

H. G. Boman. Antibacterial peptides: Basic facts and emerging concepts. Journal of Internal Medicine, 254(3):197-215, 2003. [p4,7]

D. Eisenberg. Three-dimensional structure of membrane and surface proteins. Annual Review of Biochemistry, 53:595-623, 1984. [p7, 8]

D. Eisenberg, R. M. Weiss, and T. C. Terwilliger. The helical hydrophobic moment: A measure of the amphiphilicity of a helix. Nature, 299(5881):371-374, 1982. [p7, 8]

C. D. Fjell, J. Hiss, R. E. W. Hancock, and G. Schneider. Designing antimicrobial peptides: Form follows function. Nature Reviews. Drug Discovery, 11(1):37-51, 2012. [p4]

P. Gaillard, P.-A. Carrupt, B. Testa, and A. Boudon. Molecular lipophilicity potential, a tool in 3D QSAR: Method and applications. Journal of Computer-Aided Molecular Design, 8(2):83-96, 1994. [p12]

E. Gasteiger, C. Hoogland, A. Gattiker, M. R. Wilkins, R. D. Appel, A. Bairoch, et al. Protein identification and analysis tools on the ExPASy server. In The Proteomics Protocols Handbook, pages 571-607. Springer, 2005. [p4, 5, 7]

K. Guruprasad, B. V. Reddy, and M. W. Pandit. Correlation between stability of a protein and its dipeptide composition: A novel approach for predicting in vivo stability of a protein from its primary sequence. Protein Engineering, 4(2):155-61, 1990. [p6]

R. E. W. Hancock. Cationic peptides: Effectors in innate immunity and novel antimicrobials. The Lancet. Infectious Diseases, 1(3):153-164, 2001. [p4]

T. Hessa, N. M. Meindl-Beinker, A. Bernsel, H. Kim, Y. Sato, M. Lerch-Bader, I. Nilsson, S. H. White, and G. von Heijne. Molecular code for transmembrane-helix recognition by the Sec61 translocon. Nature, 450(7172):1026-1030, 2007. [p12]

T. A. Holton, G. Pollastri, D. C. Shields, and C. Mooney. CPPpred: Prediction of cell penetrating peptides. Bioinformatics, 29(23):3094-3096, 2013. [p12]

F. Huang and W. M. Nau. A conformational flexibility scale for amino acids in peptides. Angewandte Chemie, 115(20):2371-2374, 2003. [p12]

A. Ikai. Thermostability and aliphatic index of globular proteins. Journal of Biochemistry, 88(6): 1895-1898, 1980. [p6]

S. Kawashima, P. Pokarowski, M. Pokarowska, A. Kolinski, T. Katayama, and M. Kanehisa. AAindex: Amino acid index database, progress report 2008. Nucleic Acids Research, 36(suppl 1):D202-D205, 2008. [p7]

H. Kim, J. H. Jang, S. C. Kim, and J. H. Cho. De novo generation of short antimicrobial peptides with enhanced stability and cell specificity. The Journal of Antimicrobial Chemotherapy, 69(1):1-12, 2013. [p5]

M. Kuhn. caret: Classification and Regression Training, 2014. URL http://CRAN.R-project.org/ package=caret. R package version 6.0-35. [p9]

K. Matsuzaki. Control of cell selectivity of antimicrobial peptides. Biochimica et Biophysica Acta, 1788 (8):1687-1692, 2009. [p4]

L. Monincová, J. Slaninová, V. Fučík, O. Hovorka, Z. Voburka, L. Bednárová, P. Maloň, J. Štokrová, and V. Čeřovskỳ. Lasiocepsin, a novel cyclic antimicrobial peptide from the venom of eusocial bee Lasioglossum laticeps (Hymenoptera: Halictidae). Amino Acids, 43(2):751-761, 2012. [p4]

D. S. Moore. Amino acid and peptide net charges: A simple calculational procedure. Biochemical Education, 13(1):10-11, 1985. [p6] 
S. P. Piotto, L. Sessa, S. Concilio, and P. Iannelli. YADAMP: Yet another database of antimicrobial peptides. International Journal of Antimicrobial Agents, 39(4):346-351, 2012. [p4]

P. Rice, I. Longden, and A. Bleasby. EMBOSS: The European Molecular Biology Open Software Suite. Trends in Genetics, 16(6):276-277, 2000. [p4]

J. E. Stajich, D. Block, K. Boulez, S. E. Brenner, S. A. Chervitz, C. Dagdigian, G. Fuellen, J. G. Gilbert, I. Korf, H. Lapp, et al. The Bioperl toolkit: Perl modules for the life sciences. Genome Research, 12 (10):1611-1618, 2002. [p4]

S. Thomas, S. Karnik, R. S. Barai, V. K. Jayaraman, and S. Idicula-Thomas. CAMP: A useful resource for research on antimicrobial peptides. Nucleic Acids Research, 38:D774-780, 2010. [p4]

M. Torrent, D. Andreu, V. M. Nogués, and E. Boix. Connecting peptide physicochemical and antimicrobial properties by a rational prediction model. PLoS ONE, 6(2):e16968, 2011. [p6]

G. Wang, X. Li, and Z. Wang. APD2: The updated antimicrobial peptide database and its application in peptide design. Nucleic Acids Research, 37:D933-937, 2009. [p4, 9]

M. R. Yeaman and N. Y. Yount. Mechanisms of antimicrobial peptide action and resistance. Pharmacological Reviews, 55(1):27-55, 2003. [p4, 6]

Daniel Osorio

Escuela de Biología, Facultad de Ciencias

Universidad Industrial de Santander

Colombia

daniel.osorio@correo.uis.edu.co

Paola Rondón-Villarreal

Escuela de Ingeniería Eléctrica, Electrónica y de Telecomunicaciones

Universidad Industrial de Santander

Colombia

paitorv@gmail.com

Rodrigo Torres

Escuela de Química, Facultad de Ciencias

Universidad Industrial de Santander

Colombia

rtorres@uis. edu.co 